\title{
Psychological wellbeing and physical activity in children and adolescents with inflammatory bowel disease compared to healthy controls
}

Laura MähImann ${ }^{1,4^{*}}$ (D), Markus Gerber ${ }^{3}$, Raoul I. Furlano², Corinne Legeret ${ }^{2}$, Nadeem Kalak', Edith Holsboer-Trachsler ${ }^{1}$ and Serge Brand ${ }^{1,3,5}$

\begin{abstract}
Background: Children and adolescents with inflammatory bowel disease (IBD) report impairments in daily activities, social interactions and coping. Findings regarding psychological functioning are inconsistent, while limited information is available on objectively assessed physical activity (PA). The aims of the present study were therefore to compare anthropometric dimensions, blood values, psychological functioning and PA of children and adolescents with IBD with healthy controls.

Methods: Forty-seven children and adolescents took part in the study. Of these, 23 were diagnosed with IBD (mean age: 13.88 years, 44\% females). The IBD group was divided into a medically well adjusted "remissiongroup" ( $n=14$; IBD-RE) and a group with an "active state" of disease $(n=8$; IBD-AD). Healthy controls $(n=24$; HC) were age- and gender-matched. Participants' anthropometric data, blood values and objective PA were assessed. Further, participants completed questionnaires covering socio-demographic data and psychological functioning.

Results: Participants with IBD-AD showed higher erythrocyte sedimentation rate (ESR), C-reactive protein (CRP) values, haemoglobin, and leukocyte values. IBD-AD had poorer psychological functioning and lower PA (average steps per day) compared to IBD-RE and HC. No mean differences were found between IBD-RE and $\mathrm{HC}$.

Conclusions: The pattern of results suggests that effective medical treatment of IBD in children and adolescents is associated with favorable physiological parameters, psychological dimensions and PA. Psychological counselling of children and adolescents in an active state of IBD seem to be advised in addition to standard treatment schedules.
\end{abstract}

Trial registration: NCT NCT02264275; Registered 8 October 2014.

Keywords: Inflammatory bowel diseases, Pediatrics, Anthropometric dimensions, Psychological wellbeing, Physical activity, Blood values, Healthy controls

\footnotetext{
*Correspondence: laura.maehlmann@upkbs.ch

'Psychiatric Clinics of the University of Basel, Centre for Affective, Stress and Sleep Disorders, University of Basel, Wilhelm Klein-Strasse 27, Ch-4012 Basel, Switzerland

${ }^{4}$ United Nations University - Maastricht Economic and Social Research

Institute on Innovation and Technology (UNU-MERIT), Maastricht University,

Maastricht, The Netherlands

Full list of author information is available at the end of the article
}

(c) The Author(s). 2017 Open Access This article is distributed under the terms of the Creative Commons Attribution 4.0 International License (http://creativecommons.org/licenses/by/4.0/), which permits unrestricted use, distribution, and reproduction in any medium, provided you give appropriate credit to the original author(s) and the source, provide a link to the Creative Commons license, and indicate if changes were made. The Creative Commons Public Domain Dedication waiver (http://creativecommons.org/publicdomain/zero/1.0/) applies to the data made available in this article, unless otherwise stated. 


\section{Background}

Inflammatory Bowel Disease (IBD) is a chronic, debilitating illness characterized by cycles of disease activity and quiescence. IBD is subdivided into Crohn's disease (CD), ulcerative colitis (UC) and atypical phenotypes as described by the Porto criteria (PIBD: Pediatric IBD) [1]. Although the ultimate aetiology of IBD remains unclear, three hypotheses are advanced: (1) A dysregulated inflammation emerges due to interactions between the gut luminal content (intestinal microflora) and the mucosa, especially in genetically predisposed hosts [2]. (2) Genetic factors seem to contribute slightly to the disease pathogenesis. (3) Microbial and environmental dimensions have been identified as possible contributing factors [3].

Worldwide it is estimated that $25 \%$ of all patients suffering from IBD are children and adolescents, with increasing incidence rates [4]. In children with IBD stunting of physical growth and delay in pubertal development are observed [5]. Furthermore, patients with active IBD are known to report increased erythrocytesedimentation rates (ESR) due to inflammation, decreased hemoglobin due to chronic blood loss in the gut, decreased albumin due to leaking gut at reduced intake and increased infection parameters such as leukocytes and C-reactive protein (CRP), when compared to healthy controls. Once the diagnosis is confirmed, the goals of medical therapy are to relieve symptoms, restore growth and bone health, normalize quality of life and psychosocial functioning, prevent complications and minimize the adverse effects of medications [6]. While the efficacy of anti-inflammatory pharmaceuticals are not $100 \%$ and the side effects might be severe, some young IBD patients might be in a symptom-free remission, while others are still in an active disease state. Accordingly, based on the regularly assessed PUCAI (Pediatric ulcerative colitis activity index) scores and pediatric Crohn's disease activity index (CDAI), participants in the present study were split into those with medically optimized treatment (IBD in remission, PUCAI <10; CDAI <150) the IBD - RE group, and those with not yet medically optimized treatment (active disease state), the IBD - AD group. As a result, our first hypothesis was that disease severity and the accompanying symptoms would be negatively associated with the physical development of children and adolescents with IBD. Specifically, we expected smaller and lighter body shapes and elevated inflammation markers in participants of the IBD-AD group, compared to IBD-RE and healthy controls.

In recent years, a plethora of studies have suggested that the prevalence of reduced health-related quality of life (HRQOL) and psychiatric disorders are significantly greater in young people with IBD than healthy controls [7-9]. More specifically, adolescents with IBD had higher levels of internalizing disorders such as anxiety and depression [7, 9], due to several factors such as unpredictable, unpleasant, and embarrassing symptoms, complex, demanding treatment regimens, treatment-related side effects, the ever-looming threat of exacerbations of the disease, and the need for surgical procedures in severe cases [10]. The rate of depression may be as high as 25\%, and spreads among a broad variety of psychological and social difficulties. However, as reviewed by Ross et al. [8], the evidence these conclusions are based on, were inconsistent, included few paediatric samples, and used coarsegrained psychological instruments. As a result, recognition of the importance of mental wellbeing in young IBD patients is lacking in medical practice. If these psychological conditions are left untreated, mental disorders linked to more severe IBD symptoms might emerge, as well as more frequent IBD flares, higher hospitalization rates, increased health seeking behaviour and lower compliance with treatment [11, 12]. To counter this, in the present study, validated and reliable self-rating instruments were used to assess dimensions such as psychological wellbeing, social support and peer relationships. Consequently, the second aim of the present study was to expand upon existing research by assessing children's and adolescent's psychological functioning with self-rating questionnaires, investigating specific aspects of psychological functioning, along with a depression screener.

As regards physical activity and sports participation, research showed that children and adolescents with IBD participated less in sports activities and were less fit than their counterparts without IBD [13, 14]. In the specific case of paediatric IBD, reduced physical exertion during puberty might lead to impaired bone growth, strength and density, resulting in an increased risk for osteoporosis in the long term [10]. However, a large number of studies have proven the beneficial effect of PA for a diversity of health conditions: First, PA mitigates depressive symptoms while bolstering sleep [15] and cardiovascular fitness [16]. Second, sports participation (via clubs and sports associations) has the potential to increase and deepen children's and adolescents' social skills. So far, research on PA in children and adolescents with IBD has largely been based on rough estimates. Therefore, the third aim of the present study was to assess PA behavior in children and adolescents with IBD, both subjectively using an internationally established questionnaire, and objectively with three methods: First, to investigate participants' fitness, we used the Six-Minute-WalkingTest; second, for strength assessments, we applied a hydraulic grip strength test, and third, to generate 
long-term PA reports, a FitbitFlex ${ }^{\bullet}$ accelerometer was applied.

Taking into account the state-of-the-art regarding physical development, psychological functioning and physical activity among children and adolescents with IBD, the following three hypotheses were formulated: First, following others [5, 17], we expected impaired anthropometric development among children and adolescents suffering from IBD and increased inflammatory blood markers, and particularly among those in an active state of the disease. Second, on the basis of previous research [7-9], we expected poorer psychological functioning in children and adolescents with IBD, than in their healthy counterparts, and again, particularly among those in an active state of the disease. Third, previous findings [18] led us to expect lower levels of PA (both subjective and objective) in children and adolescents with IBD than in their healthy counterparts.

\section{Methods}

Procedure

All participants were invited to the hospital to measure height, weight, waist circumference (measured $4 \mathrm{~cm}$ above the navel using a standard anthropometry tape) compared to reference values established by Taylor et al. [19] and BMI $\left(\mathrm{kg} / \mathrm{m}^{2}\right)$, which was compared to the WHO international growth references [20]. Following these anthropometric measurements, participants' blood values were collected and physical performance was examined; they also filled out a $30 \mathrm{~min}$ questionnaire with questions on psychological functioning and PA behaviour. All data were collected by two trained research assistants and supervised by a medical doctor.

The present study was approved by the local ethical committee (EKNZ: 2014:220), and was conducted in accordance to the ethical principles laid down in Declaration of Helsinki (Trial registration number: NCT02264275).

\section{Sample}

A total of 31 eligible children and adolescents with IBD were approached between April and November 2015 via the University Children's Hospital Basel (UKBB, Basel, Switzerland). Of these, 29 (93.5\%) agreed to participate in the study, though five subsequently withdrew from the study because of time constraints, and three withdraw due to acute illness (flu). The final sample consisted of 23 children and adolescents with diagnosed IBD (mean age: 13.88 years, $\mathrm{SD}=3.11,10$ females (43.5\%), $7 \mathrm{UC}, 12 \mathrm{CD}$ and $3 \mathrm{UD})$. According to the regularly assessed PUCAI (Paediatric ulcerative colitis activity index) scores and Paediatric Crohn's disease activity index (clinical scores), eight participants were in an active disease state and 14 in remission at the time of the study [11]. Inclusion criteria were as follows: (1) aged 6 to 20 years; (2) clinically and histologically confirmed diagnosis of IBD; (3) willing and able to take part in the study; (4) able to communicate and to complete questionnaires in German; (5) written informed consent from both the children/adolescents and their parents (or a legal caregiver). Exclusion criteria were: (1) severe physical diseases of the locomotor apparatus; (2) psychiatric disorders such as psychotic disorders, severe affective disorders, eating disorders, mental retardation, autism spectrum disorder; (3) unable to communicate and to complete questionnaires in German; (4) among female adolescents: pregnancy, breastfeeding, or intention to get pregnant during the study period; (5) refusal to give written informed consent. In parallel, 24 age- and gendermatched controls (mean age: 12.38 years, $\mathrm{SD}=3.24$, females $n=15(62.5 \%)$ ) were recruited by word of mouth recommendations. Inclusion criteria and exclusion criteria for healthy controls were the same as for patients with IBD except that they had to be physically healthy as reported from both participants and parents and as known from medical records. All participants were informed about the study aims and the voluntary and confidential basis of their participation. Written informed consent was signed both by participants and by their legal guardians.

Characteristics of the participants subdivided into three groups of IBD-AD, IBD-RE and healthy control are represented in Table 1.

\section{Tools}

\section{Laboratory assessment}

Using the finger prick technique, biochemical parameters were assessed involving inflammatory indices such as albumin $[\mathrm{g} / \mathrm{dl}]$, CRP [g/dl], hemoglobin $[\mathrm{g} / \mathrm{dl}]$, ESR $[\mathrm{mm} / \mathrm{h}]$, hematocrit [\%], thrombocyte $[\mathrm{g} / \mathrm{dl}]$ and leucocyte $[\mathrm{g} / \mathrm{dl}]$ count.

\section{Assessing psychological functioning}

To assess psychological functioning, participants completed the KIDSCREEN 27 [21]. The questionnaire

Table 1 Sample characteristics

\begin{tabular}{llll}
\hline & $\begin{array}{l}\text { IBD-AD } \\
(n=8)\end{array}$ & $\begin{array}{l}\text { IBD-RE } \\
(n=14)\end{array}$ & $\begin{array}{l}\mathrm{HC} \\
(n=24)\end{array}$ \\
\hline Age in years & $14.69 \pm 3.25$ & $13.23 \pm 2.96$ & $12.38 \pm 3.24$ \\
$\begin{array}{l}\text { Female } \\
\text { IBD }\end{array}$ & $4(50)$ & $6(42.9)$ & $15(62.5)$ \\
$\quad$ Ulcerative colitis & $3(37.5)$ & $4(28.6)$ & \\
$\quad \begin{array}{l}\text { Crohn's disease } \\
\text { Undefined colitis }\end{array}$ & $5(62.5)$ & $7(50)$ & \\
Time since diagnosis (in years) & $3.67 \pm 2.81$ & $4.1 \pm 2.9$ & \\
\hline
\end{tabular}

Notes: $N=46$

$I B D-A D$ IBD in an active state of the disease, IBD-RE IBD in remission, $H C$ Healthy control 
consists of 27 items covering five domains, physical wellbeing, psychological wellbeing, autonomy and relations with parents, social support and peers, and school environment. Answers were given on 5-point rating scales $(1=$ not at all, $5=$ extremely/always). Higher mean scores reflect better functioning in a specific domain. Validity has been verified by Ravens-Sieberer et al. [21] (Cronbach's $\alpha=.91$ ).

To assess symptoms of depression, the Child Depression Screener (ChilD-S) was completed [22]. The ChilD-S is a self-report screening instrument for pre-pubertal in- and out-patients in paediatric care. It consists of 8 items assessing how participants have felt for the last 2 weeks ("I am happy, I am doing fine, I feel exhausted, I worry a lot, I feel sad, I get upset quickly, I am not in the mood for anything, I often think I did something wrong"). Participants were asked to select from four alternative responses reflecting different levels of depressive symptomatology. The cut off value for clinically considerable depression is $\geq 11$, with higher values indicating more marked depressive symptoms. Validity has been verified by Frühe et al. [22] (Cronbach's $\alpha=.78)$.

\section{Assessing physical activity}

Subjective assessment The short form of the IPAQ (IPAQ-S) questionnaire was applied as an internationally approved estimator of level of PA and sedentary behaviour [23]. It provides a comparison of vigorous- and moderate-intensity PA, walking, total PA and time spent sitting on weekdays over the past seven days. PA data are reported in hours and/or minutes per day and days per week. Where minutes of intense activity exceeded 180 per day, a cut-off of 180 min per day was applied as suggested by the guidelines of the IPAQ group. Data were transformed and summed using standardized IPAQ scoring protocols to indicate total metabolic equivalent minutes (MET-minutes) of PA per week. Total METminutes per week was calculated using the following formula [23]:

$$
\begin{aligned}
& \text { [Walking MET-minutes } \left./ \text { week }=3.3^{*} \text { walking minutes }{ }^{*} \text { walking days }\right] \\
& +[\text { Moderate MET-minutes } / \text { week }=4.0 \\
& \left.{ }^{*} \text { moderate-intensity activity minutes }{ }^{*} \text { moderate days }\right] \\
& +[\text { Vigorous MET-minutes/week }=8.0 \\
& \text { * vigorous-intensity activity minutes } \left.{ }^{*} \text { vigorous-intensity days }\right] \\
& =\text { Total PA MET-minutes/week. }
\end{aligned}
$$

Furthermore, self-reported fitness was assessed on a 5point Likert scale. Validity of the instrument has been established by Hagstömer et al. [24] Sitting time was reported as the amount of time in hours and/or minutes participants usually spent sitting on a weekday during the past 7 days.

Objective assessments Grip force. Maximum isometric grip force of the dominant hand was assessed using the hydraulic hand dynamometer. Participants made three attempts. Mean outcomes were compared to reference data [25].

Functional capacity. The 6-min walking test (6 MWT), a self-paced, submaximal exercise test, was employed [26]; the test is designed to assess functional exercise capacity in patients with chronic diseases. The 6 MWT is well-standardized and is increasingly being utilized in pediatric populations with chronic diseases [27]. Walking distance is accepted as the main outcome measure of the 6 MWT. Heart rate (HR) is continuously recorded during exercise using an elastic chest strap with heart rate sensor.

Daily PA. The Fitbit-Flex (Fitbit Inc., San Francisco, CA, USA), a small and light wristband accelerometer which uses three-dimensional motion sensing technology to measure movement $24 \mathrm{~h}$ a day, was applied for a continuous assessment of 5 days (2 weekend days and 3 weekdays). It has a simple display of five LED lights which indicate the number of steps taken in a day. Fitbit data were recorded using the 1-min epoch setting and downloaded from the user website via the device's USB docking port in a raw data format. Validation studies have been conducted with children and adolescents [28].

\section{Statistical analyses}

Since normality was violated according to Shapiro-Wilk test, the groups IBD-AD, IBD-RE and $\mathrm{HC}$ were compared using the Kruskal-Wallis test with these diagnostic clusters as independent variable and anthropometric measures, psychological assessments and PA data as dependent variable. Due to heterogeneity of age distribution, Spearman's correlations were performed to determine whether significant correlations would be found beyond age and other dimensions. Accordingly, statistical calculations of anthropometric data, grip strength and $6 \mathrm{MWT}$ were controlled for age. Post-hoc tests after Whitney-U for $p$-values were performed to examine differences between the three groups. The nominal level of statistical significance was set at alpha $<.05$. Further, effect sizes were reported as partial eta-squared $\left[\eta_{p}^{2}\right]$ ) and considered as follows: small $(\mathrm{s})=.01 \leq \eta_{p}^{2} \leq .059$, medium $(\mathrm{m})=.06 \leq \eta_{p} \leq .139$, or large $\left.(\mathrm{l})=\eta_{p} \geq .14\right)$ [29]. All statistics were performed with SPSS ${ }^{\circ} 24.0$ (IBM Corporation, Armonk NY, USA) for Windows. 


\section{Results}

All descriptive and inferential statistical information is reported in the Tables and not repeated again in the written text. All Tables compare the three groups of IBD-AD, IBD-RE and HC.

\section{Anthropometric and laboratory findings}

As shown in Table 2, there was a statistically significant mean difference in thrombocytes $(p=.003)$. According to Mann-Whitney-U Post-hoc test, differences between IBD-AD and HC were significant $(p=.003)$. Further, there were no statistically significant mean differences between the three groups in the following dimensions: waist circumference, waist circumference difference from norm, height, weight to height percentage, weight, BMI, BMI z-score, percentile BMI vs age, z-score height vs age, percentile height vs age and the blood values albumin $[\mathrm{g} / \mathrm{l}]$, CRP $[\mathrm{mg} / \mathrm{l}]$, hemoglobin $[\mathrm{g} / \mathrm{l}]$, ESR $[\mathrm{mm} / \mathrm{h}$, hematocrit [\%] and leukocytes $\left[\times 10^{\wedge} 9 / 1\right]$. Investigating effect sizes, the IBD-AD group scored moderately lower on BMI z-score, percentile BMI vs age, z-score height vs age and percentile height vs age.

Further, Table 2 shows higher C-reactive protein (CRP) values, hemoglobin values, and leukocyte values in the group of IBD-AD vs IBD-RE and HC. More specifically, six (75\%) IBD-AD patients had low albumin levels, as did eight (61.54\%) IBD-RE patients and 12 (50\%) HC (reference 35 - $53 \mathrm{~g} / \mathrm{L}$ ). CRP was high in one (12.5\%) IBD-AD and one (7.1\%) IBD-RE patient (reference $<10.0 \mathrm{mg} / \mathrm{L})$. Two patients $(25 \%)$ in the IBD-AD group, two IBD-RE (14.2\%) and one control (4.2\%) were anemic with mean hemoglobin scores of $101.5 \mathrm{~g} / \mathrm{L}, 114 \mathrm{~g} / \mathrm{L}$ and $100 \mathrm{~g} / \mathrm{L}$, respectively (reference $120-160 \mathrm{~g} / \mathrm{L}$ ).

Assessing the erythrocyte sedimentation rate (ERS) one IBD-AD (12.5\%) was slightly above reference, as well as four (28.6\%) IBD-RE and six (25\%) HC (reference 4.1 $\left.-5.1 \times 10^{\wedge} 12 / \mathrm{L}\right)$. One IBD-RE $(7.1 \%)$ patient had results below reference.

Regarding hematocrit outcomes, two (25\%) IBD-AD, four (28.6\%) IBD-RE (mean 34.27\%) and three (12.5\%) HC had values below reference (36-46\%). Thrombocytosis was present in two (25\%) IBD-AD patients (reference 150 $\left.450 \times 10^{\wedge} 9 / 1\right)$. Leukocyte counts were high in two $(25 \%)$ IBD-AD, three (21.4\%) IBD-RE and one (4.2\%) HC compared to reference $\left(4.5-11 \times 10^{\wedge} 9 / 1\right)$.

\section{Psychological functioning}

As Table 3 shows, there were no statistically significant mean differences between the three groups (psychological wellbeing physical wellbeing, autonomy and parent relations,

Table 2 Anthropometrics and Blood values

\begin{tabular}{|c|c|c|c|c|c|c|c|}
\hline & \multirow{2}{*}{$\begin{array}{l}\mathrm{IBD}-\mathrm{AD} \\
(N=8) \\
M \pm S D\end{array}$} & \multirow{2}{*}{$\begin{array}{l}\text { IBD-RE } \\
(N=14) \\
M \pm S D\end{array}$} & \multirow{2}{*}{$\begin{array}{l}\mathrm{HC} \\
(N=24) \\
M \pm S D\end{array}$} & \multicolumn{4}{|c|}{ Statistical Analysis } \\
\hline & & & & $H$ & $p$ & np2 & $\begin{array}{l}\text { Interpretation } \\
\text { of effect size }\end{array}$ \\
\hline Waist circumference $(\mathrm{cm})$ & $67.63 \pm 9.04$ & $65.65 \pm 7.17$ & $64.48 \pm 8.29$ & 0.384 & 0.823 & 0.01 & $\mathrm{~S}$ \\
\hline Difference from norm & $-9.81 \pm 6.15$ & $-8.68 \pm 4.95$ & $-8.82 \pm 6.0$ & 0.521 & 0.783 & 0 & S \\
\hline Height (cm) & $153.48 \pm 17.53$ & $152.69 \pm 12.02$ & $152.26 \pm 18.0$ & 0.212 & 0.903 & 0 & s \\
\hline Weight to height (\%) & $44.08 \pm 3.07$ & $42.94 \pm 2.13$ & $42.77 \pm 3.49$ & 1.108 & 0.585 & 0.02 & s \\
\hline Weight (kg) & $45.28 \pm 15.85$ & $42.79 \pm 12.83$ & $44.37 \pm 15.75$ & 0.215 & 0.899 & 0 & $\mathrm{~s}$ \\
\hline $\mathrm{BMI}$ & $18.6 \pm 3.37$ & $17.91 \pm 2.78$ & $18.36 \pm 3.16$ & 0.074 & 0.965 & 0.12 & $\mathrm{~m}$ \\
\hline BMI z-score & $-0.588 \pm 0.79$ & $-0.34 \pm 0.95$ & $-0.19 \pm 0.87$ & 1.368 & 0.511 & 0.1 & $\mathrm{~m}$ \\
\hline Percentile BMI vs Age & $31.98 \pm 24.31$ & $39.82 \pm 26.23$ & $43.89 \pm 26.43$ & 1.386 & 0.516 & 0.1 & $\mathrm{~m}$ \\
\hline z-score Height vs Age & $-0.911 \pm 1.73$ & $0.02 \pm 0.86$ & $0.19 \pm 1.01$ & 2.234 & 0.334 & 0.1 & $\mathrm{~m}$ \\
\hline Percentile Height vs Age & $32.04 \pm 32.99$ & $52.91 \pm 25.71$ & $54.83 \pm 31.53$ & 2.236 & 0.333 & 0.07 & $\mathrm{~m}$ \\
\hline Albumin $[\mathrm{g} / \mathrm{l}]$ & $23 \pm 21.94$ & $19.32 \pm 19.20$ & $20.79 \pm 18.76$ & 0.767 & 0.686 & 0 & $\mathrm{~S}$ \\
\hline CRP $[\mathrm{mg} / \mathrm{l}]$ & $3.02 \pm 4.56$ & $3.28 \pm 5.69$ & $0.71 \pm 0.85$ & 4.159 & 0.125 & 0.13 & m \\
\hline Hemoglobin [g/l] & $139.25 \pm 8.62$ & $134.92 \pm 16.26$ & $135.86 \pm 12.33$ & 1.423 & 0.493 & 0.01 & $\mathrm{~s}$ \\
\hline $\mathrm{ESR}[\mathrm{mm} / \mathrm{h}]$ & $4.83 \pm 0.36$ & $4.87 \pm 0.54$ & $4.88 \pm 0.36$ & 0.281 & 0.874 & 0 & s \\
\hline Hematocrit [\%] & $39.4 \pm 2.11$ & $38.09 \pm 3.99$ & $38.75 \pm 3.17$ & 0.359 & 0.837 & 0.02 & $\mathrm{~s}$ \\
\hline Thrombocyts $\left[\times 10^{\wedge} 9 / 1\right]$ & $323.25 \pm 55.61$ & $302.58 \pm 56.15$ & $271.55 \pm 46.54$ & 11.211 & 0.003 & 0.13 & $\mathrm{~m}$ \\
\hline Leukocyten $[\times 10 \wedge 9 / l]$ & $10.16 \pm 2.01$ & $9.28 \pm 2.83$ & $8.25 \pm 1.29$ & 4.088 & 0.13 & 0.11 & $\mathrm{~m}$ \\
\hline
\end{tabular}

Notes: $N=46$, degrees of freedom always $=2,41, p<.05$ statistically significant; effect sizes: small $(\mathrm{s})=.01>\eta \mathrm{np} 2<.059$, medium $(\mathrm{m})=.06>\eta \mathrm{n}<.139$, or large $(\mathrm{I})=n \mathrm{p} \geq .14$

$I B D-A D$ IBD in an active state of the disease, IBD-RE IBD in remission, $H C$ Healthy Control, Waist circumference norm references is Taylor et al. [19], $B M I$ Body Mass Index; CRP C-reactive protein, ESR Erythrocyte Sedimentation Rate 
Table 3 Psychological functioning and depression

\begin{tabular}{|c|c|c|c|c|c|c|c|}
\hline & \multirow{2}{*}{$\begin{array}{l}\text { IBD-AD } \\
(N=8) \\
M \pm S D\end{array}$} & \multirow{2}{*}{$\begin{array}{l}\text { IBD-RE } \\
(N=14) \\
M \pm S D\end{array}$} & \multirow{2}{*}{$\begin{array}{l}H C \\
(N=24) \\
M \pm S D\end{array}$} & \multicolumn{4}{|c|}{ Statistical Analysis } \\
\hline & & & & $H$ & $p$ & пр2 & Interpretation of effect sizes \\
\hline \multicolumn{8}{|l|}{ Kidsscreen-27: } \\
\hline Physical Wellbeing & $17.71 \pm 3.59$ & $18.5 \pm 3.61$ & $19.38 \pm 3.16$ & 3.740 & 0.155 & 0.04 & s \\
\hline Psychological Wellbeing & $28.14 \pm 5.61$ & $31.86 \pm 2.28$ & $31.46 \pm 2.41$ & 3.258 & 0.197 & 0.16 & । \\
\hline Parent \& Autonomy & $31.14 \pm 3.53$ & $31.57 \pm 3.17$ & $31.479 \pm 2.95$ & 0.238 & 0.89 & 0 & s \\
\hline Peers \& Social Support & $16.71 \pm 2.43$ & $17.43 \pm 2.38$ & $17.29 \pm 2.24$ & 0.705 & 0.719 & 0.01 & s \\
\hline School Environment & $15.57 \pm 3.41$ & $17.07 \pm 1.86$ & $17.29 \pm 2.35$ & 1.033 & 0.599 & 0.06 & m \\
\hline ChilD-S & $6.75 \pm 5.78$ & $4.07 \pm 3.29$ & $3.75 \pm 2.75$ & 1.895 & 0.395 & 0.09 & $\mathrm{~m}$ \\
\hline
\end{tabular}

Notes: $N=46$; degrees of freedom always $=2.41 ; \mathrm{p}<.05$ statistically significant; effect sizes: $\mathrm{small}(\mathrm{s})=.01>\mathrm{np} 2<.059$. medium $(\mathrm{m})=.06>n \mathrm{p}<.139$. or large $(\mathrm{I})=n \mathrm{p} \geq .14$

$I B D-A D$ IBD in an active state of the disease, IBD-RE IBD in remission, HC Healthy Control, ChilD-S Child Depression Screener

social support and peers, school experience). Descriptively, IBD-AD had lower scores on the psychological wellbeing (PWB) dimension of the KIDSCREEN-27, while the other two groups did not differ from each other. The school dimension $(\mathrm{SCH})$ showed a medium effect of .063 , reflecting lower scores in the IBD-AD group.

Results of the depression scale indicated a medium effect size of .092, with the highest depression scores in the IBD-AD group and no differences between IBD-RE and HC.

\section{Physical activity}

Table 4 shows that results on a subjective scale assessing the last 7 days, the IPAQ questionnaire did not indicate any statistically significant mean differences in self-reported PA between the three groups IBD$A D, I B D-R E$ and HC. On a descriptive level, the IBD$\mathrm{AD}$ group reported more vigorous physical activity (medium ES of .088) than the IBD-RE and HC groups. However, self-estimated fitness was lowest in the IBD-AD group $(E S=.109)$.

Table 4 Subjective and objective physical activity

\begin{tabular}{|c|c|c|c|c|c|c|c|}
\hline & \multirow{2}{*}{$\begin{array}{l}\text { IBD-AD } \\
(N=8) \\
M \pm S D\end{array}$} & \multirow{2}{*}{$\begin{array}{l}\text { IBD-RE } \\
(N=14) \\
M \pm S D\end{array}$} & \multirow{2}{*}{$\begin{array}{l}H C \\
(N=24) \\
M \pm S D\end{array}$} & \multicolumn{4}{|c|}{ Statistical Analysis } \\
\hline & & & & $H$ & $p$ & пр2 & $\begin{array}{l}\text { Interpretation } \\
\text { of effect sizes }\end{array}$ \\
\hline MET min vigorous & $2610 \pm 2745.99$ & $2202.86 \pm 1832.39$ & $1378.33 \pm 1023.34$ & 2.124 & 0.355 & 0.09 & $\mathrm{~m}$ \\
\hline MET min moderate & $710 \pm 789.5$ & $557.14 \pm 406.85$ & $1198.33 \pm 2032.29$ & 0.594 & 0.752 & 0.04 & s \\
\hline MET min walking & $315.56 \pm 136.19$ & $321.16 \pm 390.79$ & $397.79 \pm 330.5$ & 1.878 & 0.409 & 0.02 & s \\
\hline Total MET & $3635.56 \pm 2819.76$ & $3081.16 \pm 1994.34$ & $2974.45 \pm 2354.31$ & 0.546 & 0.768 & 0.01 & s \\
\hline Sitting Weekend & $382.5 \pm 198.84$ & $407.14 \pm 193.25$ & $351.33 \pm 163.72$ & 0.504 & 0.779 & 0.02 & s \\
\hline Sitting Weekday & $411.25 \pm 132.23$ & $408.21 \pm 143.41$ & $384.38 \pm 107.55$ & 1.022 & 0.608 & 0.01 & s \\
\hline Fitness & $2.25 \pm 1.17$ & $2.93 \pm 0.92$ & $3.08 \pm 0.78$ & 3.501 & 0.177 & 0.11 & $\mathrm{~m}$ \\
\hline Heart Rate pre $6 \mathrm{MWT}$ & $94.63 \pm 15.91$ & $101.69 \pm 13.19$ & $94.26 \pm 11.25$ & 3.357 & 0.189 & 0.11 & $\mathrm{~m}$ \\
\hline Heart Rate post 6 MWT & $142.88 \pm 46.41$ & $161.92 \pm 28.19$ & $163.13 \pm 28.9$ & 0.623 & 0.74 & 0.05 & s \\
\hline Heart Rate increase & $48.25 \pm 45.99$ & $60.23 \pm 26.47$ & $68.87 \pm 30.35$ & 0.801 & 0.679 & 0.07 & $\mathrm{~m}$ \\
\hline 6 MWT Distance & $655.38 \pm 135.69$ & $719.08 \pm 84.91$ & $687.78 \pm 88.02$ & 1.247 & 0.544 & 0.07 & $\mathrm{~m}$ \\
\hline Borg 1-10 & $4 \pm 1.31$ & $4.31 \pm 2.72$ & $3.74 \pm 2.4$ & 0.689 & 0.715 & 0.02 & s \\
\hline Grip strength (kg) & $28.72 \pm 11.61$ & $24.54 \pm 8.66$ & $24.12 \pm 9.64$ & 0.877 & 0.65 & 0.02 & s \\
\hline Difference from norm & $1.42 \pm 9.34$ & $-3.2 \pm 8.25$ & $-2.08 \pm 7.36$ & 0.837 & 0.663 & 0.03 & s \\
\hline Average daily steps & $8049 \pm 3614$ & $10.689 \pm 3089$ & $12.473 \pm 4248$ & 5.923 & 0.049 & 0.18 & I \\
\hline
\end{tabular}

Notes: $N=46$. degrees of freedom always $=2.41 ; \mathrm{p}<.05$ statistically significant; effect sizes: $\mathrm{small}(\mathrm{s})=.01>\eta \mathrm{p} 2<.059$. $\mathrm{medium}(\mathrm{m})=.06>\mathrm{np}<.139$. or large $(\mathrm{I})=n \mathrm{p} \geq .14$

IBD-AD IBD in an active state of the disease, IBD-RE IBD in remission, HC Healthy Control, MET Metabolic Equivalent, 6 MWT 6-min walking test 
Mean grip strength did not differ statistically significantly between the three groups IBD-AD, IBD-RE and HC.

Even though statistically non-significant, the mean $6 \mathrm{MWT}$ results indicated descriptive differences, with IBD-AD patients achieving the shortest distance compared to IBD-RE and $\mathrm{HC}$ (medium ES=.070). The IBD-AD also had less increase in heart rate (medium ES =.073), but still rated the intensity of the test as high as the other two groups (small ES =.018).

For the objective step counts as measured by the FitbitFlex ${ }^{\oplus}$, a statistical significant mean difference $(p=.049)$ and a large effect size of .183 were observed; the average count was 8049 steps per day in the IBD-AD group, 10,689 steps per day in IBD-RE and 12,473 steps per day in the HC group. According to Mann-Whitney-U post-hoc tests, comparisons between groups were not significant.

\section{Discussion}

The key findings of the present study were that children and adolescents with IBD-AD had poorer psychological functioning than children and adolescents with IBD-RE and HC. Furthermore, they had a lower functional capacity (6MWT) and engaged less in objectively assessed physical activity (average steps per day) compared to children and adolescents with IBD in remission or ageand gender-matched healthy controls. The present pattern of results adds to the current literature in an important way, showing that medically well-adjusted children and adolescents with IBD do not differ from healthy controls with regard to psychological functioning and objective and subjective PA.

Three hypotheses were tested and each of these is considered now in turn.

Our first hypothesis was that there would be anthropometric differences; specifically, we expected that participants with IBD were smaller, lighter and to have greater inflammation, especially among the more severe cases of IBD. Our data partly supported this hypothesis. The IBD-AD group had a moderately lower BMI z-score, BMI percentile, z-score height for age and percentile height for age when compared to both other groups. Therefore, the present data are in accordance with previous findings $[14,30]$. In general, $10-40 \%$ of children with IBD are affected by growth failure [14, 30] and impaired nutritional status [31]. Underlying reasons are anorexia, malabsorption, intestinal inflammation and corticosteroid usage [32]. Given that the data available do not allow a deeper understanding of the pattern of results, we speculate that current treatment strategies lead to good disease control and less growth retardation [33].

With the first hypothesis, we also expected to detect indicators for inflammation and disease-related influences in the blood values of IBD patients. This hypothesis was partially supported; scores for the inflammatory markers ESR, CRP and leukocytes were higher among those with IBD-AD. Two patients with active IBD and two in remission were anemic, as well as one participant in the control group. In general, chronic anemia is prevalent and rapidly recurring in patients with IBD [34]. On the other hand, iron deficiency is a well-known phenomenon among many healthy female teenagers [35]. The small reported differences can be accounted to constant monitoring of blood values by the treating physician, who provides the patients with either oral or intravenous iron supplementation as soon as necessary. Looking at albumin values, it is not unusual to observe lower values in patients with IBD, as indicated by our findings. Patients with IBD might lose albumin due to increased gut permeability, even during symptom-free episodes [36]. However, we were unable to explain the low albumin values found in half of the healthy control participants. Common underlying reasons are undernourishment [37], decreased production due to liver disease [38] and increased excretion due to kidney problems [39], factors, which however could be ruled out by the supervising health professionals in the present study. Finally, the increased average hemoglobin among IBD-AD in comparison to $\mathrm{HC}$ was very surprising. The only possible explanation might be a blood clotting during blood sample taking since the hematocrit is elevated as well.

With the second hypothesis, we expected poorer psychological functioning in children and adolescents with IBD-AD compared to IBD-RE and healthy controls, and data did partially support this. Children and adolescents with increased disease activity had poorer psychological functioning in the area of psychological wellbeing. On the flipside, no other dimension of psychological functioning (physical functioning, autonomy and parent relations, social support and peers, and school experience) showed significant differences across the three groups, IBD-AD, IBD-RE and HC. Therefore, the present data do not match the study by Herzer et al. [40], showing a lower overall HRQOL among patients with IBD. Further, the present data do not accord with those studies reporting impaired physical functioning [41], impaired family functioning [40], limited participation in social activities [7], lower emotional functioning [42], and problematic school experience and performance.

Previous evidence can be explained by the association between increased disease activity and lower psychological functioning. One may claim that greater disease severity might be accompanied by an increasing presence of disruptive gastrointestinal symptoms and abdominal pain, which in turn need to be treated with more robust and invasive methods. Such increasing disease burden might result in psychological distress [43]. Two further hypotheses are the inflammation-depression hypothesis and the brain gut hypothesis. These two 
hypotheses claim that the increased production of proinflammatory cytokines (TNF alpha) is known to affect the brain both, directly and indirectly, thereby increasing symptoms of depression $[44,45]$. Further, there is a bidirectional relationship, since psychological stress in turn increases the likelihood of inflammation, which again increases the occurrence of depressive symptoms [42]. The pro-inflammatory effect of experimental stress has been confirmed in human studies, and at the same time, inflammatory markers have been found to be raised in depressed patients [46]. To a wider extent, the inflammatory, unpredictable and disruptive nature of severe IBD, if not properly treated, could lead to an increase in internalizing symptoms (e.g., anxiety and depression). In particular, given that the long-term course of the disease is characterized by progressive deterioration [47], young patients might be at increased risk for psychological distress.

While comparing children and adolescents in remission with the healthy control group, we did not find differences on any of the HRQOL dimensions. This can be attributed to the fact that children in remission are by definition symptom-free [11]. A recent publication by the SWISS IBD Cohort even reported higher psychological functioning in IBD patients than in controls. The authors attributed this unexpected finding to the excellent social support in the young patients' environment [41]. Walter et al. [48], on the basis of a careful examination of the literature, noted that older studies (1989 2004) reported higher depression rates; subsequent advances in treatment may be responsible for the lower levels of psychological distress observed more recently. Therefore, we suggest that screening and treatment for mental wellbeing should be implemented especially for those with increased disease activity and potentially weaker social support.

Our third hypothesis, following others [18, 49], was that physical activity levels (subjective and objective) would be lower in children and adolescents with IBD than in their healthy counterparts. While the three groups did not differ regarding subjectively assessed PA in our study, lower objectively assessed PA was indicated by the number of steps per day, especially in the IBDAD group. Thus, the present data are in account with previous results, however based on objective measurements. Werkstetter et al. [14] also reported reduced amounts of steps per day in children and adolescents with IBD, while studies of adult patients have found that suffering from IBD tended to lead to a sedentary lifestyle [50]. An explanation might be that patients with active IBD are restricted and discouraged by unpredictable symptoms, physical restriction, inconvenience and discomfort [49]. Furthermore, we found that children and adolescence with active IBD perceived physical strain to be more vigorous as compared to IBD-RE and HC. This is reflected in their subjectively estimated greater intensity of the $6 \mathrm{MWT}$, while achieving less distance than the other groups and their extremely elevated selfreported vigorous PA levels over the last 7 days. This finding indicated a lower fitness in children and adolescents with active IBD, and was in line with findings of Ploeger et al. [13] Children with IBD exhibited impaired aerobic and anaerobic exercise capacity, compared to reference values.

However, a lower amount of PA might be an issue for the following reasons. Generally, regular low intensity exercise is beneficial in reducing distress and improving quality of life in young patients with IBD [51]. A UK survey revealed that PA made patients with IBD feel better and healthier, boosted energy, reduced IBD symptoms, provided an alternative focus and fostered feelings of normality [49]. On a physiological level, recent findings indicated that IBD patients could experience antiinflammatory effects from the myokines released during skeletal muscle contraction while exercising, thereby inhibiting the release of protective heat shock proteins (Hsps), which help to regulate inflammation and immunity [52]. It was further asserted that PA is an anabolic stimulus, reducing inflammation and positively affecting growth factors, i.e., IGF-I [53]. Additionally, Robbins et al. [49] argued that regular PA should be undertaken in IBD to help maintain bone mineral density and prevent osteoporosis. We should note, however, that exaggerated amounts of (high-intensity) PA might lead to an increase in symptoms. Vigorous PA such as distance running and endurance exercise commonly might cause gastrointestinal discomfort including nausea, heartburn, and even gastrointestinal bleeding in patients with IBD [54]. By contrast, moderate PA seems to be useful in improving many aspects of the lives of patients with IBD. Nonetheless, a fear of symptoms exacerbation may be an indication that patients lack the impetus to start PA, and clinicians may be reluctant to prescribe PA, even though it might serve as a protective and preventive factor with respect to IBD. Collectively, we claim that regular moderate PA should receive greater attention in scientific intervention studies as adjuvant to prescribed pharmacotherapies.

Despite the novelty of the findings and the application of internationally validated and accepted questionnaires and objective measures, several limitations warrant against an overgeneralization of the present findings. First, the relatively small sample, especially the small number of children in an active disease state, created difficulties in detecting statistically significant effects. Though we also relied on effect sizes, which are not sensitive to sample size. Second, the recruitment of the sample was restricted to the German speaking part of 
Switzerland. Of these patients, only participants willing and able to participate were assessed. Even though we tried to cover an extensive range of factors, it was not possible to control for all confounders, such as microbial or environmental conditions, which might have influenced two or more outcomes in the same or in opposite directions. In this context, one suggestion for future studies might be the assessment of objective disease severity, fecal calprotectin as another objective measure. Otherwise we would suggest extending the cross sectional design to include lifestyle intervention studies and their supportive effect in the disease coping process. Last, we did not distinguish between patients with $\mathrm{CD}$ and $\mathrm{UC}$; whereas for diagnostic reasons such an approach would have been easy to follow and justify, we decided to split patients into IBD-AD and IBD-RE, as suggested by recent research such as Reigada et al. [42, 55].

\section{Conclusion}

The pattern of results suggests that effective medical treatment of IBD in children and adolescents is associated with favorable physiological parameters, psychological dimensions and PA. Psychological counselling of children and adolescents with severe IBD seem to be advised in addition to standard treatment schedules.

\section{Abbreviations \\ 6 MWT: Six minute walking test; CD: Crohn's disease; CDAl: Crohn's disease activity index; ChilD-S: Child Depression Screener; CRP: C-reactive protein; ERS: Erythrocyte sedimentation rate; ES: Effect size; HC: Healthy control; HR: Heart rate; HRQOL: Health related quality of life; Hsps: Heat shock proteins; IBD: Inflammatory bowel diseases; IBD-AD: IBD in an active state of the disease; IBD-RE: IBD in remission; MET: Metabolic equivalent; PA: Physical activity; PIBD: Pediatric IBD; PUCA: Pediatric ulcerative colitis activity index; UC: Undefined colitis}

\section{Acknowledgements}

We thank the Kantonsspital Aarau for providing additional recruitment options. We are also grateful to Noe Stoll for support during data collection and data entry. Finally, we thank Nick Emler (Surrey, UK) for proofreading the manuscript and Harald Seelig for supporting the statistical analysis.

\section{Funding}

We thank the Freiwillige Akademische Gesellschaft Basel (FAG, Basel, Switzerland) for financially supporting the project.

\section{Availability of data and materials}

The datasets used and/or analysed during the current study available from the corresponding author on reasonable request.

\section{Authors' contributions}

Conception and Design, LM, MG, RIF, CL, NK, EHT, SB; Acquisition of data, LM, M.G, RIF, CL, NK; Analysis and interpretation of data: LM, MG, RF, CL, NK, EHT, $S B$; Drafting of manuscript: $L M, M G, R I F, C L, N K$, EHT, SB. All authors read and approved the final manuscript.

\section{Ethics approval and consent to participate}

The present study was approved by the local ethical committee (Ethikkommission Nordwest- und Zentralschweiz (EKNZ): 2014:220), and was also conducted in accordance to the ethical principles laid down in Declaration of Helsinki (Trial registration number: NCT02264275). Voluntary participation was encouraged and informed consent to participate in the study was obtained from each patient. All participants and their legal guardian had to consent the study participation in written format.

Patient records or information was anonymized and de-identified prior to analysis.

\section{Consent for publication}

Not applicable,

Competing interests

The authors declare that they have no competing interests.

\section{Publisher's Note}

Springer Nature remains neutral with regard to jurisdictional claims in published maps and institutional affiliations.

\section{Author details}

${ }^{1}$ Psychiatric Clinics of the University of Basel, Centre for Affective, Stress and Sleep Disorders, University of Basel, Wilhelm Klein-Strasse 27, Ch-4012 Basel, Switzerland. ${ }^{2}$ Pediatric Gastroenterology \& Nutrition, University Children's Hospital Basel, Basel, Switzerland. ${ }^{3}$ Department of Sport, Exercise and Health, Sport Science Section, University of Basel, Basel, Switzerland. ${ }^{4}$ United Nations University - Maastricht Economic and Social Research Institute on Innovation and Technology (UNU-MERIT), Maastricht University, Maastricht, The Netherlands. ${ }^{5}$ Substance Abuse Prevention Research Center; Sleep Disorders Research Center, Psychiatry Department, Kermanshah University of Medical Sciences, Kermanshah, Iran.

Received: 5 September 2016 Accepted: 30 November 2017

Published online: 12 December 2017

\section{References}

1. Levine A, de Bie Cl, Turner D, et al. Atypical disease phenotypes in pediatric ulcerative colitis: 5-year analyses of the EUROKIDS registry. Inflamm Bowel Dis. 2013;19:370-7

2. Lemberg DA, Day AS. Crohn disease and ulcerative colitis in children: an update for 2014. J Paediatr Child Health. 2015:51:266-70.

3. Loddo I, Romano C. Inflammatory bowel disease: genetics, epigenetics, and pathogenesis. Front Immunol. 2015;6 Available from: http://journal. frontiersin.org/Article/10.3389/fimmu.2015.00551/abstract. Cited 21 Mar 2016

4. Braegger CP, Ballabeni P, Rogler D, Vavricka SR, Friedt M, Pittet V, et al. Epidemiology of inflammatory bowel disease: is there a shift towards onset at a younger age? J Pediatr Gastroenterol Nutr. 2011;53:141-4.

5. Pappa H, Thayu M, Sylvester F, Leonard M, Zemel B, Gordon C. Skeletal health of children and adolescents with inflammatory bowel disease. J Pediatr Gastroenterol Nutr. 2011;53:11-25.

6. Rosen MJ, Dhawan A, Saeed SA. Inflammatory bowel disease in children and adolescents. JAMA Pediatr. 2015;169:1053.

7. Engstrom I. Mental-health and psychological functioning in children and adolescents with inflammatory bowel-disease: a comparison with children having other chronic illnesses and with healthy-children. J Child Psycho Psychiatry. 1992;3:563-82.

8. Ross SC, Strachan J, Russell RK, Wilson SL. Psychosocial functioning and health related quality of life in paediatric inflammatory bowel disease: a systematic review. J Pediatr Gastroenterol Nutr. 2011;53:480.

9. Vaisto T, Aronen ET, Simola P, Ashorn M, Kolho KL. Psychosocial symptoms and competence among adolescents with inflammatory bowel disease and their peers. Inflamm Bowel Dis. 2010;1:27-35.

10. Rabizadeh S, Dubinsky M. Update in pediatric inflammatory bowel disease. Rheum Dis Clin N Am. 2013;39:789-99.

11. Mikocka-Walus A, Knowles SR, Keefer L, Graff L. Controversies revisited: a systematic review of the comorbidity of depression and anxiety with inflammatory bowel diseases. Inflamm Bowel Dis. 2016:22:752-62.

12. Spekhorst LM, Hummel TZ, Benninga MA, van Rheenen PF, Kindermann A. Adherence to oral maintenance treatment in adolescents with inflammatory bowel disease. J Pediatr Gastroenterol Nutr. 2016;62:264-70.

13. Ploeger H, Obeid J, Nguyen T, Takken T, Issenman R, de Greef M, et al. Exercise and inflammation in pediatric Crohn's disease. Int J Sports Med. 2012;33:671-9.

14. Werkstetter KJ, Ullrich J, Schatz SB, Prell C, Koletzko B, Koletzko S. Lean body mass, physical activity and quality of life in paediatric patients 
with inflammatory bowel disease and in healthy controls. J Crohns Colitis. 2012;6:665-73.

15. Loprinzi PD, Cardinal BJ. Association between objectively-measured physical activity and sleep, NHANES 2005-2006. Ment. Health and Phys Act. 2011;4:65-9.

16. Gerber $M$, Lindwall $M$, Lindegård $A$, Börjesson $M$, Jonsdottir $H$. Cardiorespiratory fitness protects against stress-related symptoms of burnout and depression. Patient Educ Couns. 2013;93:146-52.

17. Hummel TZ, Tak E, Maurice-Stam H, Benninga MA, Kindermann A, Grootenhuis MA. Psychosocial developmental trajectory of adolescents with inflammatory bowel disease. J Pediatr Gastroenterol Nutr. 2013;57:219-24.

18. Chan D, Robbins H, Rogers S, Clark S, Poullis A. Inflammatory bowel disease and exercise: results of a Crohn's and Colitis UK survey. Frontline Gastroenterol. 2014:5:44-8.

19. Taylor RW, Jones IE, Williams SM, Goulding A. Evaluation of waist circumference, waist-to-hip ratio, and the conicity index as screening tools for high trunk fat mass, as measured by dual-energy X-ray absorptiometry, in children aged 3-19 y. Am J Clin Nutr. 2000;72:490-5.

20. WHO Multicentre Growth Reference Study Group. WHO Child growth standards: length/height-for-age, weight-for-age, weight-for-length, weightfor-height and body mass index-for-age: methods and development. Geneva: World Health Organization; 2006.

21. Ravens-Sieberer U, Herdman M, Devine J, Otto C, Bullinger M, Rose M, et al. The European KIDSCREEN approach to measure quality of life and wellbeing in children: development, current application, and future advances. Qual Life Res. 2014;23:791-803.

22. Frühe B, Allgaier A-K, Pietsch K, Baethmann M, Peters J, Kellnar S, et al. Children's Depression Screener (ChilD-S): development and validation of a depression screening instrument for children in pediatric care. Child Psychiatry Hum Dev. 2012;43:137-51.

23. IPAQ. Guidelines for data processing and analysis of the International Physical Activity Questionnaire (IPAQ) - short and long forms, revised on November 2005. 2005. Available from: http://www.ipaq.ki.se/scoring.pdf. Cited 15 Mar 2010

24. Hagströmer M, Bergman P, De Bourdeaudhuij I, Ortega FB, Ruiz JR, Manios Y, et al. Concurrent validity of a modified version of the International Physical Activity Questionnaire (IPAQ-A) in European adolescents: the HELENA study. Int J Obes. 2008;32:S42-8.

25. Mathiowetz V, Wiemer DM, Federman SM. Grip and pinch strength: norms for 6-to 19-year-olds. Am J Occup Ther. 1986;40:705-11.

26. Solway S, Brooks D, Lacasse Y, Thomas S. A qualitative systematic overview of the measurement properties of functional walk tests used in the cardiorespiratory domain. Chest. 2001;119:256-70.

27. Hassan J, van der Net J, Helders PJM, Prakken BJ, Takken T. Six-minute walk test in children with chronic conditions. Br J Sports Med. 2010;44:270-4.

28. Meltzer LJ, Hiruma LS, Avis K, Montgomery-Downs H, Valentin J. Comparison of a commercial accelerometer with polysomnography and actigraphy in children and adolescents. Sleep. 2015:38:1323-30.

29. Cohen J. Statistical power analysis for the behavioral sciences. 2nd ed. Hillsdale: N.J: Routledge; 1988.

30. Dubinsky M. Special issues in pediatric inflammatory bowel disease. World J Gastroenterol. 2008:14:413.

31. Gasparetto M. Crohn's disease and growth deficiency in children and adolescents. World J Gastroenterol. 2014:20:13219.

32. Shamir R. Nutritional aspects in inflammatory bowel disease. J Pediatr Gastroenterol Nutr. 2009:48:S86-8.

33. De Greef E, Hoffman I, Smets F, Van Biervliet S, Bontems P, Hauser B, et al. Paediatric Crohn's disease: disease activity and growth in the BELCRO cohort after 3 years follow-up. J Pediatr Gastroenterol Nutr. 2016;63:253.

34. Kulnigg S, Teischinger L, Dejaco C, Waldhör T, Gasche C. Rapid recurrence of IBD-associated anemia and iron deficiency after intravenous iron sucrose and erythropoietin treatment. Am J Gastroenterol. 2009;12:1460-7.

35. Looker AC, Dallman PR, Carroll MD, Gunter EW, Johnson CL. Prevalence of iron deficiency in the United States. JAMA. 1997;12:973-6.

36. Michielan A, D'Incà R. Intestinal permeability in inflammatory bowel disease: pathogenesis, clinical evaluation, and therapy of leaky gut. Mediat Inflamm. 2015:2015:628157.

37. Vermeire S, Van Assche G, Rutgeerts P. Laboratory markers in IBD: useful, magic, or unnecessary toys? Gut. 2006;55:426-31.
38. Bernardi M, Maggioli C, Zaccherini G. Human albumin in the management of complications of liver cirrhosis. Crit Care. 2012;16:211.

39. Birn $H$, Christensen El. Renal albumin absorption in physiology and pathology. Kidney Int. 2006:69:440-9.

40. Herzer M, Denson LA, Baldassano RN, Hommel KA. Patient and parent psychosocial factors associated with health-related quality of life in pediatric inflammatory bowel disease. J Pediatr Gastroenterol Nutr. 2011;52:295-9.

41. Rogler D, Fournier N, Pittet $V$, Bühr P, Heyland $K$, Friedt $M$, et al. Coping is excellent in Swiss children with inflammatory bowel disease: results from the Swiss IBD cohort study. J Crohns Colitis. 2014;8:409-20.

42. Goodhand JR, Wahed M, Mawdsley JE, Farmer AD, Aziz Q, Rampton DS Mood disorders in inflammatory bowel disease: relation to diagnosis, disease activity, perceived stress, and other factors. Inflamm Bowel Dis. 2012;18:2301-9.

43. Gray WN, Denson LA, Baldassano RN, Hommel KA. Disease activity, behavioral dysfunction, and health-related quality of life in adolescents with inflammatory bowel disease. Inflamm Bowel Dis. 2011;17:1581-6.

44. Raison CL, Capuron L, Miller AH. Cytokines sing the blues: inflammation and the pathogenesis of depression. Trends Immunol. 2006;27:24-31.

45. van den Brink G, Stapersma L, El Marroun H, Henrichs J, Szigethy EM, Utens EM, et al. Effectiveness of disease-specific cognitive-behavioural therapy on depression, anxiety, quality of life and the clinical course of disease in adolescents with inflammatory bowel disease: study protocol of a multicentre randomised controlled trial (HAPPY-IBD). BMJ Open Gastroenterol. 2016;3:e000071.

46. Miller $\mathrm{AH}$, Raison $\mathrm{CL}$. The role of inflammation in depression: from evolutionary imperative to modern treatment target. Nat Rev Immunol. 2016;16:22-34.

47. Pittet $V$, Juillerat $P$, Mottet C, Felley C, Ballabeni P, Burnand B, et al. Cohort profile: the Swiss Inflammatory Bowel Disease Cohort Study (SIBDCS). Int J Epidemiol. 2009;38:922-31.

48. Walter JG, Kahn SA, Noe JD, Schurman JV, Miller SA, Greenley RN. Feeling fine: anxiety and depressive symptoms in youth with established IBD. Inflamm Bowel Dis. 2016;22:402-8.

49. Robbins H, Poullis A, Rogers S. Inflammatory bowel disease and exercisepreliminary results of a Crohn's and colitis UK survey. Gastroenterol Today. 2012;22:62-3.

50. Narula N, Fedorak RN. Exercise and inflammatory bowel disease. Can J Gastroenterol. 2008;22:497-504.

51. Ng V, Millard W, Lebrun C, Howard J. Low-intensity exercise improves quality of life in patients with Crohn's disease. Clin J Sport Med. 2007;17:384-8.

52. Chen $Y$, Noble EG. Is exercise beneficial to the inflammatory bowel diseases? An implication of heat shock proteins. Med Hypotheses. 2009;72:84-6.

53. Sanderson IR. Growth problems in children with IBD. Nat Rev Gastroenterol Hepatol. 2014;11:601

54. Colditz GA, Cannuscio CC, Frazier AL. Physical activity and reduced risk of colon cancer: implications for prevention. Cancer Causes Control. 1997:8:649-67.

55. Reigada LC, Hoogendoorn CJ, Walsh LC, Lai J, Szigethy E, Cohen BH, et al. Anxiety symptoms and disease severity in children and adolescents with Crohn disease. J Pediatr Gastroenterol Nutr. 2015;60:30-5.

\section{Submit your next manuscript to BioMed Central and we will help you at every step:}

- We accept pre-submission inquiries

- Our selector tool helps you to find the most relevant journal

- We provide round the clock customer support

- Convenient online submission

- Thorough peer review

- Inclusion in PubMed and all major indexing services

- Maximum visibility for your research

Submit your manuscript at www.biomedcentral.com/submit 